\title{
Erratum: Antibacterial properties of nanoparticles
}

[Trends in Biotechnology 30 (2012), 499-511]

\section{Mohammad J. Hajipour ${ }^{1}$, Katharina M. Fromm ${ }^{2}$, Ali Akbar Ashkarran ${ }^{3}$, Dorleta Jimenez de Aberasturi ${ }^{4,5}$, Idoia Ruiz de Larramendi ${ }^{5}$, Teofilo Rojo ${ }^{5}$, Vahid Serpooshan ${ }^{6}$, Wolfgang J. Parak ${ }^{4}$, and Morteza Mahmoudi ${ }^{1,7}$}

\footnotetext{
${ }^{1}$ Laboratory of NanoBio Interactions, Department of Nanotechnology, Faculty of Pharmacy, Tehran University of Medical Sciences, Tehran, Iran

${ }^{2}$ University of Fribourg, Department of Chemistry, Chemin du Musée 9, CH-1700 Fribourg, Switzerland

${ }^{3}$ Department of Physics, Faculty of Basic Sciences, University of Mazandaran, Babolsar, Iran

${ }^{4}$ Fachbereich Physik and WZMW, Philipps Universität Marburg, Marburg, Germany

${ }^{5}$ Department of Inorganic Chemistry, UPV/EHU, Bilbao, Spain

${ }^{6}$ Division of Pediatric Cardiology, Department of Pediatrics, Stanford University School of Medicine, Stanford, CA 94305-5101, USA

${ }^{7}$ Nanotechnology Research Center, Faculty of Pharmacy, Tehran University of Medical Sciences, Tehran, Iran
}

Figures 3 and 4 contained errors. The corrected figures and figure captions are shown here. The authors apologize for this error.

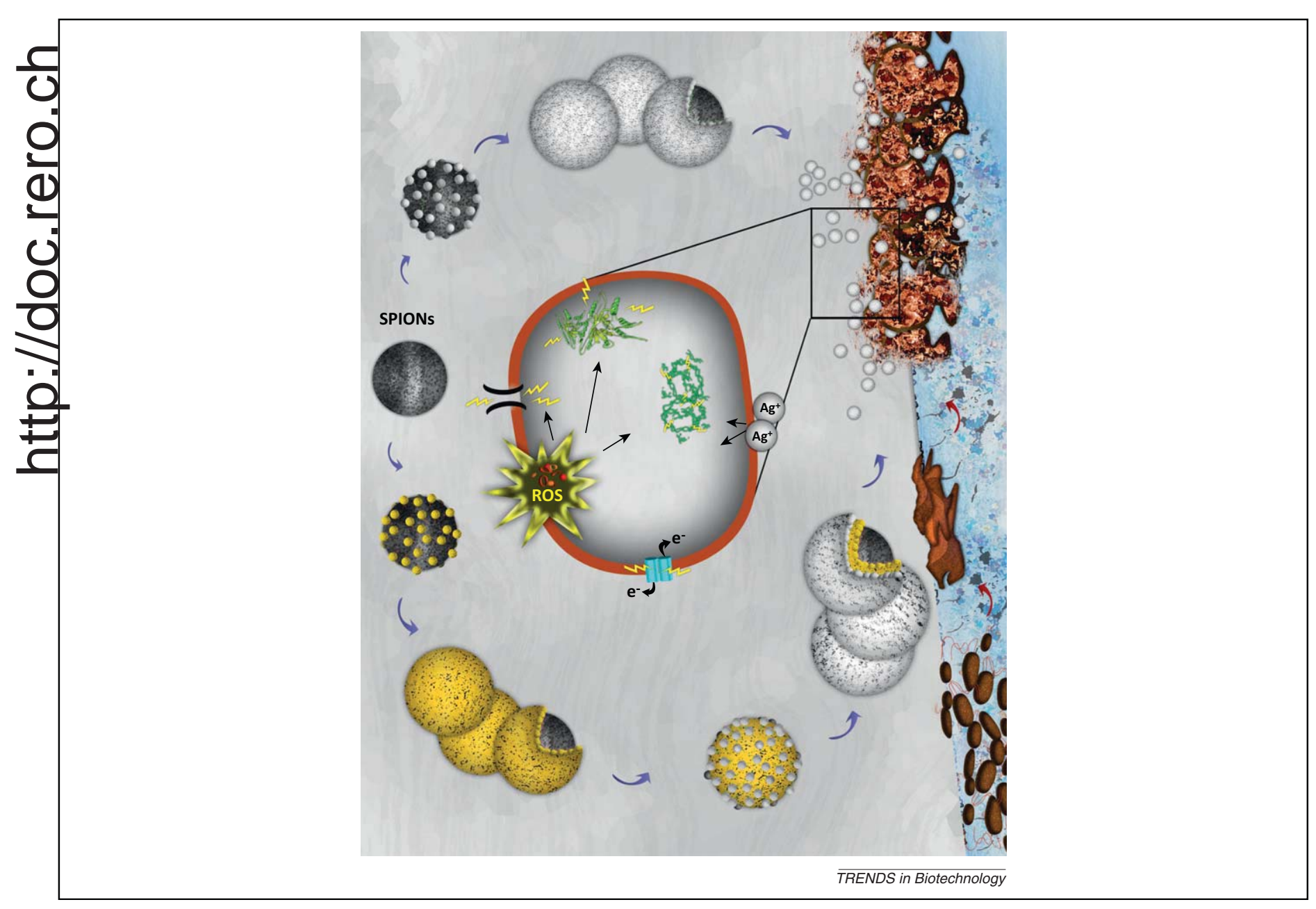

Figure 3. Schematic representation of toxicology effect of multifunctional nanoparticles (NPs) in bacterial biofilms. Monodisperse superparamagnetic iron oxide NPs (SPIONs; black spheres) are coated with silver (gray shell), gold (yellow shell), and silver ring-coated, gold-coated SPIONs; silver ring-coated SPIONs and silver ring-coated, gold-coated SPIONs have strong toxic effects on bacterial biofilms, by penetration into the biofilms. Both SPIONs cores and the intermediate gold shell have the capability to induce heat by applying alternative magnetic and laser fields, respectively; the produced heat can be used as additional means to escalate bacterial death using these NPs. The magnified section in the center illustrates the irreversible effects of NPs and their ions on the various parts of the bacteria.

\footnotetext{
DOI of original article: http://dx.doi.org/10.1016/j.tibtech.2012.06.004.

Corresponding author: Mahmoudi, M. (Mahmoudi@biospion.com,

Mahmoudi-M@TUMS.ac.ir, Mahmoudi@Illinois.edu)
} 


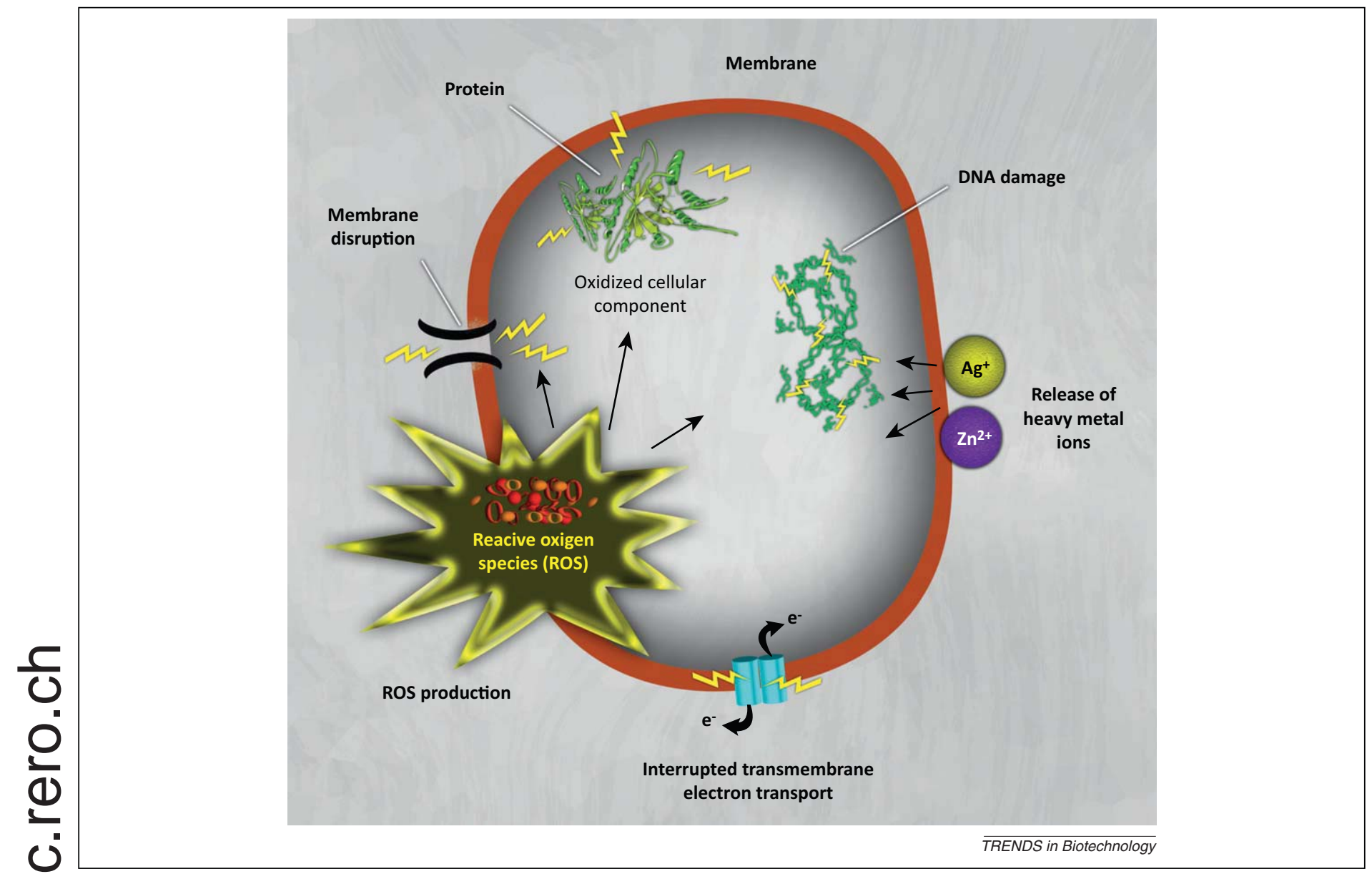

Figure 4. Mechanisms of toxicity of nanoparticles (NPs) against bacteria. NPs and their ions (e.g., silver and zinc) can produce free radicals, resulting in induction of oxidative stress (i.e., reactive oxygen species; ROS). The produced ROS can irreversibly damage bacteria resulting in bacterial death.

0167-7799/\$ - see front matter ( 2012 Elsevier Ltd. All rights reserved.

http://dx.doi.org/10.1016/j.tibtech.2012.10.007 Trends in Biotechnology, January 2013, Vol. 31 , No. 1 